\title{
Editorial
}

\section{Why biventricular pacing might be of value in refractory heart failure?}

Ten years ago, an Austrian group proposed implanting dual chamber pacemakers in advanced heart failure patients who did not meet the usual criteria for a pacing indication. ${ }^{1}$ Short term results were encouraging. A revolutionary idea was born: using cardiac pacing as an adjuvant therapy to medical treatment in drug refractory heart failure.

Meanwhile, however, pharmacological treatment made considerable progress. Angiotensin converting enzyme (ACE) inhibitors, $\beta$ blockers, and spironolactone have significantly reduced mortality and morbidity in New York Heart Association (NYHA) class II-IV patients, while improving their quality of life..$^{2-4}$ But that benefit is probably not permanent and will be limited in time. A variety of non-pharmacological approaches are available to treat these refractory heart failure patients. Heart transplant remains the best solution but it can only be applied to a restricted number of patients. So, for more than 10 years now, permanent dual chamber pacing with short atrioventricular delay has been proposed as an adjuvant treatment of advanced heart failure; however, the initially encouraging results were not proved in a long term follow up during prospective studies. ${ }^{56}$ One of the main causes for the failure of standard dual chamber pacing is probably that in patients with chronic left ventricular (LV) dysfunction, although it corrects, at least partially, atrioventricular asynchrony of the left heart, it also enhances the electromechanical consequences of intraventricular conduction delay which are often found in this type of patient.

Indeed, prolonged PR interval and wide QRS are frequently observed in patients with chronic heart failure associated with LV systolic dysfunction. Wilensky and colleagues thus demonstrated that atrioventricular and intraventricular conduction disorders, with $30 \%$ mean increase in PR interval and QRS duration, had been gradually occurring in more than $80 \%$ of patients who died from pathologically proven dilated cardiomyopathy (DCM), over a mean follow up period of 30 months. $^{7}$ Other studies revealed a non-negligible prevalence of intraventricular conduction delay (as defined by QRS duration $>120 \mathrm{~ms}$ ) in chronic heart failure patients estimated at $27-53 \%{ }^{8}$ These conduction abnormalities-intraventricular conduction delay in particular - are considered to be an independently predictive factor of mortality. Thus in the VEST (vesnarinone trial) study, the six year mortality rate in patients with chronic heart failure with altered LV function (LV ejection fraction $<40 \%$ ) was significantly higher in patients whose QRS duration exceeded $110 \mathrm{~ms}$ (65\%) than in those where it did not $(40 \%)$, regardless of the degree of LV impairment. ${ }^{9}$

In addition, these conduction disorders have deleterious effects both on systolic function and on LV filling, and they can induce or enhance mitral "functional" regurgitation. Xiao and colleagues demonstrated, in DCM patients, that the presence of a left bundle branch block (LBBB) was associated with a more than $80 \%$ increase in $\mathrm{LV}$ pre-ejection contraction time and a $60 \%$ increase in LV relaxation time; there was also a negative correlation between the QRS duration and the $+\mathrm{dP} / \mathrm{dt}$. So, the wider the QRS, the lower the contractility. ${ }^{10}$ In the same studies, analysing the parameters of LV diastolic function revealed that the LV filling time was significantly reduced (by nearly $40 \%$ ) in DCM patients in the presence of LBBB or a significant prolongation of the PR interval (>200 ms). In parallel, the quality of atrial contribution to LV filling was impaired, as reflected on transmitral Doppler by a single phase flow linked to $\mathrm{E}$ wave and A wave superimposition. ${ }^{11}$ Lastly, in patients with LV systolic dysfunction, the presence of an LBBB or prolonged PR interval is associated with an increase in the duration of mitral valve regurgitation. Incidentally, the presence of an LV-left atrial diastolic gradient - frequently found in patients with DCM and atrioventricular conduction disorders - can be the origin of diastolic mitral regurgitation.

Standard dual chamber pacing with single site ventricular pacing applied to the right ventricular apex permits extending the LV filling time and increasing, at least in appearance, the atrial contribution to ventricular filling, but the price to pay is an aggravation of intra- and interventricular desynchronisation. Other right ventricular pacing sites - the right ventricular outflow tract in particular-were evaluated but induced no additional benefit. ${ }^{12}$ Only direct His bundle pacing, which preserves the sequence of intrinsic activation, appears to be associated with a significant and durable improvement of LV function parameters, but that method is technically delicate and can only be used in patients with normal HisPurkinje activation, as indicated by a spontaneously narrow QRS complex. ${ }^{13}$ This highly selective population does not match those patients considered for biventricular multisite pacing.

The aim of multisite biventricular pacing is to correct not only the atrioventricular asynchrony but also the nonuniformity of ventricular activation, contraction and relaxation sequences. It was proposed primarily to patients with drug refractory heart failure with LV systolic dysfunction and wide QRS complex. The first implantations in man were simultaneously performed in 1994 by two teams, in a restricted number of patients, and results were rather encouraging. ${ }^{14} 15$

Based on these preliminary experiments, several groups evaluated this concept in acute haemodynamic studies. The first one was conducted immediately after coronary artery bypass surgery in 18 patients. ${ }^{16}$ Biventricular cardiac pacing significantly improved cardiac index and systemic vascular resistance, as compared with no pacing. Cazeau and colleagues confirmed these results in eight patients with advanced heart failure ${ }^{15}$ Our group showed that in 18 class III/IV patients with mean (SD) QRS duration of 170 (36) $\mathrm{ms}$, biventricular DDD pacing with individually optimised atrioventricular synchrony significantly improved cardiac index $(+35 \%)$ and pulmonary capillary wedge pressure $(-19 \%)$ in relation to baseline. ${ }^{17}$ Furthermore, biventricular pacing was significantly more effective than 
right ventricular DDD pacing, regardless of the pacing site. Other groups, during acute haemodynamic studies, assessed the effects of LV pacing alone, by comparison with biventricular pacing. Blanc and colleagues showed that LV free wall (LVFW) pacing increased systemic blood pressure and decreased mean pulmonary capillary pressure in the same proportions as biventricular pacing. ${ }^{18}$ These data were confirmed by Aurrichio and colleagues ${ }^{19}$ and Kass and associates ${ }^{20}$ who demonstrated that LVFW-VDD pacing alone was slightly more effective than biventricular VDD pacing, whereas right ventricular VDD pacing brought about no benefit.

This trend to a greater acute haemodynamic benefit during LVFW-VDD pacing has to be interpreted with caution, however. Because of the mean programmed value of optimal atrioventricular delay used in these two studies (125 ms), the effect of LVFW-VDD pacing was mainly the result of a fusion beat between intrinsic conduction, activating the right ventricle and part of the septum precociously, and pre-excitation of LVFW by the pacemaker. It is unlikely that such an optimal fusion could be maintained during permanent pacing because of the evolution of atrioventricular conduction (natural trend for the PR interval to prolong, effect of drugs, etc). In contrast, we can assume that biventricular VDD pacing, which results in permanent and complete capture from the two ventricular sites, will provide a more stable activation pattern.

These latter two studies also revealed a very significant fact, which will probably help in selecting the best potential responder patients: all the responder patients had a baseline QRS duration above $150 \mathrm{~ms}$ whereas it was below $150 \mathrm{~ms}$ in non-responder patients. These studies also demonstrated that haemodynamic improvement depended more on the ventricular pacing sites than on the quality of atrioventricular resynchronisation.

In view of the encouraging results noted during acute haemodynamic studies, permanent biventricular pacing has enjoyed rapid development, but was it justified? The functional beneficial results of early open studies appear to support such hopes and encourage doctors to go further and faster. However, a number of questions remain. Who are the appropriate candidates for multisite biventricular pacing? What inclusion criteria should be used to assess ventricular dysynchronisation (electrical, mechanical, or both)? Should both ventricles be paced simultaneously, or will LV pacing be sufficient? Where should the left ventricle be paced? On one site only or on several sites? It must be borne in mind that despite recent technological advances, implanting leads for $\mathrm{LV}$ pacing remains a delicate technique.

But beware, trying to move too fast can only be detrimental to this new treatment. Biventricular pacing should still be considered as an experimental treatment in the evaluation process. Results from ongoing prospective and randomised studies will be instructive, in particular those of the crossover European MUSTIC (multisite stimulation in cardiomyopathy) trial which should be reported soon. If the technique can be validated in terms of functional benefit and quality of life, then its impact on mortality, morbidity, and cost-effectiveness will have to be assessed. The various drug classes all went through such steps before being acknowledged as valid treatments of heart failure. Multisite cardiac pacing must go the same route.

C LECLERCQ J-C DAUBERT

Département de Cardiologie et Maladies Vasculaires,

Centre Cardio-Pneumologique,

Hopital Pontchaillou,

rue Henri Le Guilloux,

35033 Rennes Cedex 09, France

email: christophe.leclercq@chu-rennes.fr

1 Hochleitner $\mathrm{M}$, Hortnagl $\mathrm{H}$, Choi-Keung $\mathrm{Ng}$, et al. Usefulness of physiologic dual-chamber pacing in drug-resistant idiopathic dilated cardiomyopathy. Am f Cardiol 1990;66:198-202.

2 CONSENSUS Trial Study Group. Effects of enalapril on mortality in severe congestive heart failure. Results of the north Scandinavian enalapril survival study. N Engl f Med 1987;316:1429-35.

3 Bristow M. $\beta$-adrenergic receptor blockade in chronic heart failure. Circulation 2000;101:558-69.

4 The Randomized Aldactone Evaluation Study Investigators. The effect of spironolactone on morbidity and mortality in patients with severe heart failure N Engl f Med 1999;341:709-17.

5 Brecker SJ, Xiao HB, Sparrow J, et al. Effects of dual-chamber pacing with short atrioventricular delay in dilated cardiomyopathy. Lancet 1992;340: 1308-11.

6 Linde C, Gadler F, Edner M, et al. Results of atrioventricular synchronous pacing with optimized delay in patients with severe congestive heart failure. Am f Cardiol 1995;75:919-23.

7 Wilensky RL, Yudelman P, Cohen AI, et al. Serial electrocardiographic changes in idiopathic dilated cardiomyopathy confirmed at necropsy. $A m \mathcal{F}$ Cardiol 1988;62:276-83.

8 Aaronson K, Schwartz S, Chen T, et al. Development and prospective validation of a clinical index to predict survival in ambulatory patients referred for cardiac transplant evaluation. Circulation 1997;95:2660-7.

9 Venkateshawar K, Gottipaty K, Krelis P, et al for the VEST investigators. The resting electrocardiogram provides a sensitive and inexpensive marker of prognosis in patients with chronic congestive heart failure [abstract]. $\mathcal{F}$ Am Coll Cardiol 1999;33:145A

10 Xiao H, Roy C, Fujimoto S, et al. Natural history of abnormal conduction and its relation to prognosis in patients with dilated cardiomyopathy. Int $\mathcal{F}$ Cardiol 1996;53:163-70.

11 Nishimura RA, Hayes DL, Holmes DR Jr, et al. Mechanism of hemodynamic improvement by dual-chamber pacing for severe left ventricular dysfunction: an acute Doppler and catheterization study. $\mathcal{F} \mathrm{Am}$ Coll Cardiol 1995;25:281-8.

12 Victor F, Leclercq C, Mabo P, et al. Optimal right ventricular pacing site in chronically implanted patients: a propective randomized cross-over comparison of apical and outflow tract pacing. 7 Am Coll Cardiol 1999;33: 311-6.

13 Deshmukh P, Casavant DA, Romanyshyn M, et al. Permanent direct His bundle pacing, a novel approach to cardiac pacing in patients with normal His-Purkinje activation. Circulation 2000;101:869-77.

14 Bakker P, Meijburg H, De Jonge, et al. Beneficial effects of biventricular pacing in congestive heart failure [abstract]. PACE 1994;17:820A

15 Cazeau S, Ritter P, Lazarus A, et al. Multisite pacing for end-stage heart failure: early experience. PACE 1996;19(pt II):1748-57.

16 Foster AH, Gold MR, McLaughlin JS. Acute hemodynamic effects of atriobiventricular pacing in humans. Ann Thorac Surg 1995;59:294-300.

17 Leclercq C, Cazeau S, Le Breton H, et al. Acute hemodynamic effects of biventricular DDD pacing in patients with end-stage heart failure. $\mathcal{F} \mathrm{Am}$ Coll Cardiol 1998;32:1825-31.

18 Blanc JJ, Etienne Y, Gilard M, et al. Evaluation of different ventricular pacing sites in patients with severe heart failure. Circulation 1997;96:3273-7.

19 Kass $\mathrm{Da}$, Chen $\mathrm{CH}$, Curry $\mathrm{C}$, et al. Improved left ventricular mechanics from acute VDD pacing in patients with dilated cardiomyopathy and ventricular conduction delay. Circulation 1999;99:1567-73.

20 Aurrichio A, Stellbrink C, Block M, et al. Effect of pacing chamber and atrioventricular delay on acute systolic function of paced patients with congestive heart failure. Circulation 1999;99:2993-3001. 